\title{
HISTOLOGICAL STUDIES OF DENTINE USING VAN GIESON AND GOMORI TRICHOME DYES
}

M. Mohd. Bakri, A. Mat Salleh. Histological studies of dentine using Van Gieson and Gomori trichome dyes. Annal Dent Univ Malaya 2003; 10: 6-10.

\begin{abstract}
Decalcified permanent teeth were sectioned and stained with Van Gieson and Gomori trichome dyes. Sections dyed with the Van Gieson dye did not produce any zones in dentine but with the Gomori trichome dye, four different zones of dentine were produced. Zone 1 begins at the predentine-dentine junction while zone 4 ends at the enamel - dentine junction. In zone 1 , the intertubular dentine was stained clearly while in zone 3 intense staining of the peritubular dentine was observed. The result of this study supports the previous findings reported by other workers that the formation of intertubular dentine takes place in zone 1 while peritubular dentine occurs in zone 3 .
\end{abstract}

Key words: histology, van Gieson, Gomori trichome, histochemical staining, zonation of dentine.

\section{INTRODUCTION}

Dentinogenesis involves formation of a predentine phase which is followed by the formation of the inorganic phase at the mineralization front (1). The molecular basis underlying the mineralization process associated with the conversion of predentine to dentine is poorly understood. However, there is evidence that indicates areas rich in mucoplysaccharides are associated with the mineralization process (2). It has been shown dyes such as Alcian blue are effective for detecting acid mucopolysaccharides (3) while the Periodic Acid Schiff (PAS) dye for detecting polysaccharides $(4,5)$.

It has been found, histologically that dentine can be differentiated into 3 or 4 zones $(4,5,6)$. Zone 1 is referred to as the junction between dentine and predentine and is $20-25 \mathrm{~mm}$ wide. Adjacent to zone 1 is zone 2 which has a thickness of $40-80 \mathrm{~mm}$ wide. Zone 3 is situated external to zone 2 and there is variation as to the width of this zone as this was the last zone described by Symons (5) where else Mjor (4) has described an additional zone, zone 4. Zone 4 represents the major portion of dentine which includes the outermost region of the dentine - enamel junction.

Through out the entire thickness of dentine or in the zonations of dentine as previously described, the intertubular dentine takes up a different stain from the peritubular dentine. It has been shown that the intertubular dentine stained well in zone 1 and the

\section{Original Article}

\author{
M. Mohd. Bakril, A. Mat Salleh² \\ JLecturer \\ 2Private Practitioner \\ JDepartment of Oral Biology, \\ Faculty of Dentistry, University of Malaya. \\ 50603 Kuala Lumpur, Malaysia. \\ 2Klinik Pergigian Tiew, PT 4772A, \\ Tingkat 1, Jln PS-1/19, Taman Semarak Satu, \\ Nilai, Negeri Sembilan. \\ Corresponding author - Marina Mohd. Bakri
}

staining was markedly reduced while in zone $2(4,5)$. This seems to suggest that the formation of intertubular dentine is taking place in zone 1 and that there is a rapid fall in the activities of the intertubular dentine in zone 2. On the other hand, peritubular dentine was found to be stained well in zone 3, indicating that formation of this type of dentine is taking place in zone 3. Both peritubular and intertubular dentine are separate entity and the formation of intertubular and peritubular dentine occurs separately, with intertubular dentine forming first followed by peritubular dentine (7). Weiner et al have shown that there was structural continuity between peritubular and intertubular dentine and that the principal protein of peritubular dentine was different from that of intertubular dentine (8). This paper attempts to study the histology of dentine using the Van Gieson and Gomori trichome dyes.

\section{MATERIALS AND METHOD}

Fifteen freshly extracted tooth (incisors and molars) from adults of 20 - 70 years of age were collected for use in this study. The caries-free teeth were obtained immediately after extraction from patients treated in the Oral and Maxillofacial Surgery Department, Faculty of Dentistry, University of Malaya. The teeth were fixed in neutral buffered formalin for one day before demineralisation by $15 \%$ Ethylene iamine Tetraacetic Acid (EDTA) for $6-8$ weeks. The solution of EDTA must be changed every 2 days within this period.

The end point of demineralisation was determined by radiography of the specimen. Tissues were then processed through graded alcohols, xylene and finally embedded in paraffin wax in Tissue Tek cassettes and moulds. With the use of the microtome (lung RM 2035, Leica), 3mm sections were obtained from paraffin wax embedded blocks of each tooth. 
Following deparaffinization in xylene and hydrated through $100 \%$ ethanol, the sections were washed thoroughly in deionised water for 10 minutes. The sections were then stained according to the methods specific for Van Gieson and Gomori trichome staining respectively. For Van Gieson staining, slides were immersed in celestine blue for 3 minutes, washed in water before being placed in alum haematoxylin for 3 minutes. Slides were repeatedly washed in water before being placed in $1 \%$ acid alcohol. A final wash with water was done before staining in Van Gieson's solution for 5 minutes. Slides were dehydrated, cleared and mounted in DPX

The method for Gomori trichome staining included immersing the slides in Haematoxylin for 1 minute followed by washing of slides in water. Slides were then stained with the Gomori trichome staining solution for approximately 30 seconds to 1 minute. A final rinse in $0.2 \%$ acetic acid was done before the slides were dehydrated, cleared and mounted in DPX.

\section{RESULTS}

The predentine was lightly stained by both the dyes used in this study. With the Van Gieson dye, no zonation of dentine was evident (Fig. 1) but for Gomori trichome, 4 zones of dentine were present. In zone 1, the intertubular dentine was stained green (Fig. 2) while in zone 2 (Fig. 3), the intertubular dentine appeared light red. The peritubular dentine was stained clearly in zone 3 (Fig. 4) where it appeared green in colour. The intertubular dentine maintain its red colour until the fourth zone. The fourth zone appeared red as there are less tubules in this zone (Fig. 4).

\section{DISCUSSION}

Although there was no zonation of dentine with the Van Gieson dye, it was observed that the intertubular dentine near the predentine region was clearly stained. This region may also be referred as zone 1 as it has been described previously that the area in zone 1 comprise of the junction between dentine and predentine with a width of $20-25 \mathrm{~mm}(4,5,6)$. The staining of intertubular dentine in zone 1 was also reported when other types of dyes were used $(4,5)$ while studies carried out by Mjor (4) using the Masson's trichome dye showed no zonations of dentine.

In this study, Gomori trichome produces 4 zonations of dentine and in zone 1, it was observed that the staining was almost entirely produced by intertubular matrix and there was no evidence of staining in the peritubular matrix. Therefore, the staining of the intertubular dentine in zone 1 or in the region nearest to the predentine seems to confirm that the formation of intertubular dentine is rapidly taking place in zone 1. Histochemical stains such as Alcian Blue and Toluidine blue has been used to demonstrate the distribution of acid mucopolysaccharides in tissues (4,5). A high content of acid mucopolysaccharides is often found in areas where calcification is taking place. In addition, the intense staining observed when PAS was used (9) further suggested that the presence of some additional polysaccharide apart from the present acid mucopolysaccharide was also present in zone 1 . These facts suggested that active changes associated with calcification is taking place rapidly in zone 1 and areas rich in mucopolysaccharides have been shown to be linked to the calcification process of dentine (2).

Although the formation of dentine from predentine is not well understood, it is thought that the noncollagenous protein is involved in the mineralization process. Light microscope immunoperoxidase staining (10) demonstrated the presence of stainable proteoglycans in predentine and dentine. However, there was a definite difference between the types of proteoglycan found in predentine and dentine. Goldberg (11) showed the presence of glycoaminosglycans at the dentine surface of the predentine-dentine surface and indicated that glycoaminoglycans may play a role as a nucleator agent. In a different study, Goldberg (12) who used stains that retained proteoglycans revealed a 1-2 micron wide intermediary zone of predentine at the dentine predentine border but the staining was found to be better on the dentine side then on predentine.

The reduced staining of the intertubular dentine in zone 2 by the Gomori trichome dye may indicate that the intertubular dentine, once formed, may undergo very little change and this has also been reported in studies using the histochemical dyes $(4,5)$ where the amount of mucopolysaccharide as well as other types of polysaccharide seems to be reduced in zone 2 than in zone 1 .

In this study, the effect of the Gomori trichome dye on the intertubular dentine in zone 3 is similar to that in zone 2. Therefore it may confirm previous findings that very little change is taking place within the intertubular matrix of zone 3 once the intertubular dentine has been formed. It has been shown that the peritubular dentine was clearly stained and this zone has been described as the most distinct zone by Mjor (4) because of its relationship to the development of the highly mineralized peritubular dentine.

The peritubular dentine first appears about 60 $100 \mathrm{~mm}$ from the dentine - predentipe junction $(5,13)$. The proteins of peritubular dentine was stained with Stains All dye and the principal protein of the peritubular dentine has high molecular weight and a different amino acid composition than that of the intertubular dentine (8). The peritubular dentine was shown to be more highly calcified than the intertubular dentine, confirming the microradiographic observations of Blake (13 ) and Miller (14). The staining of the peritubular dentine by stains such as Alcian Blue $(4,5)$ 


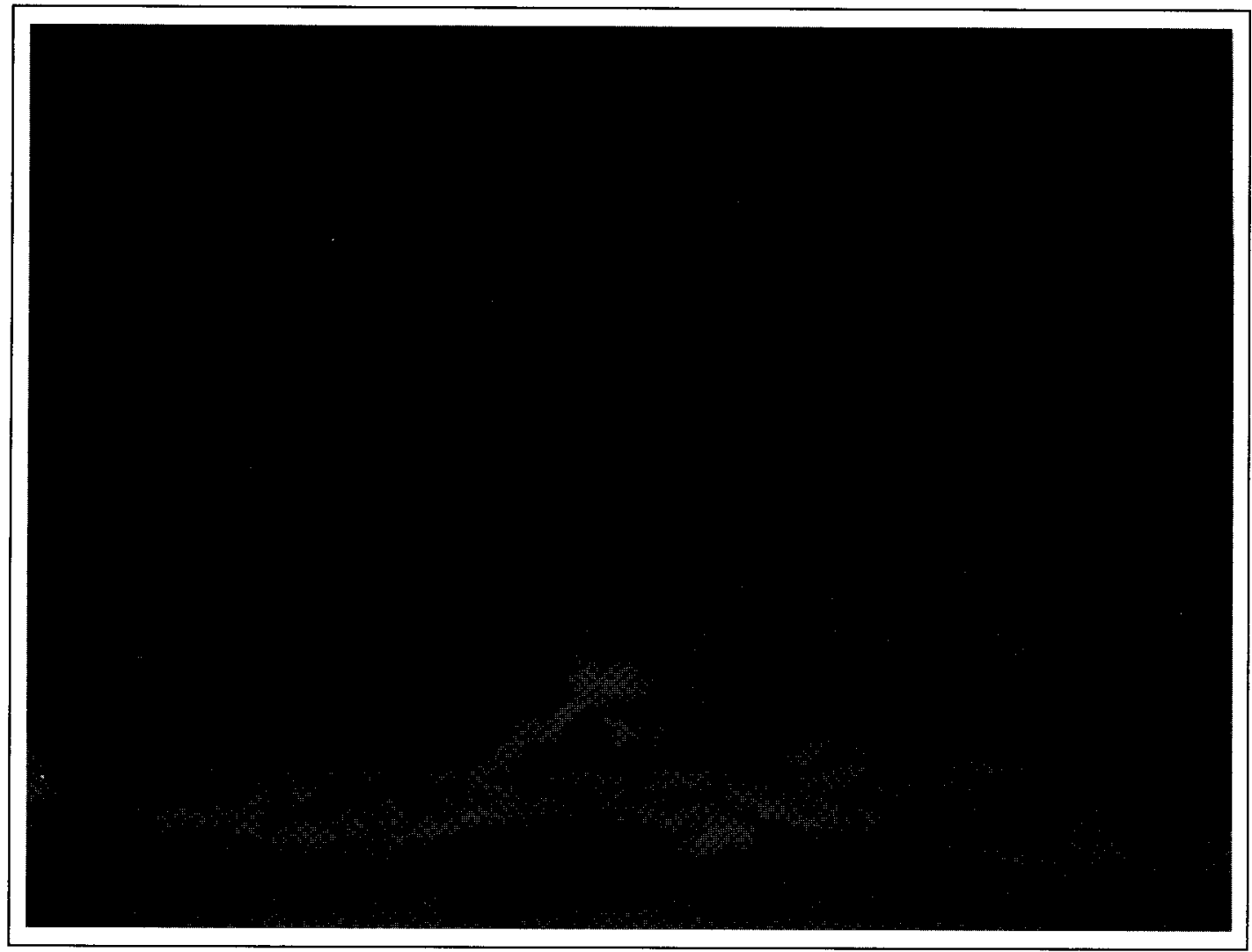

Figure 1: Van Gieson stained section. No zonation of dentine was present. (X2)

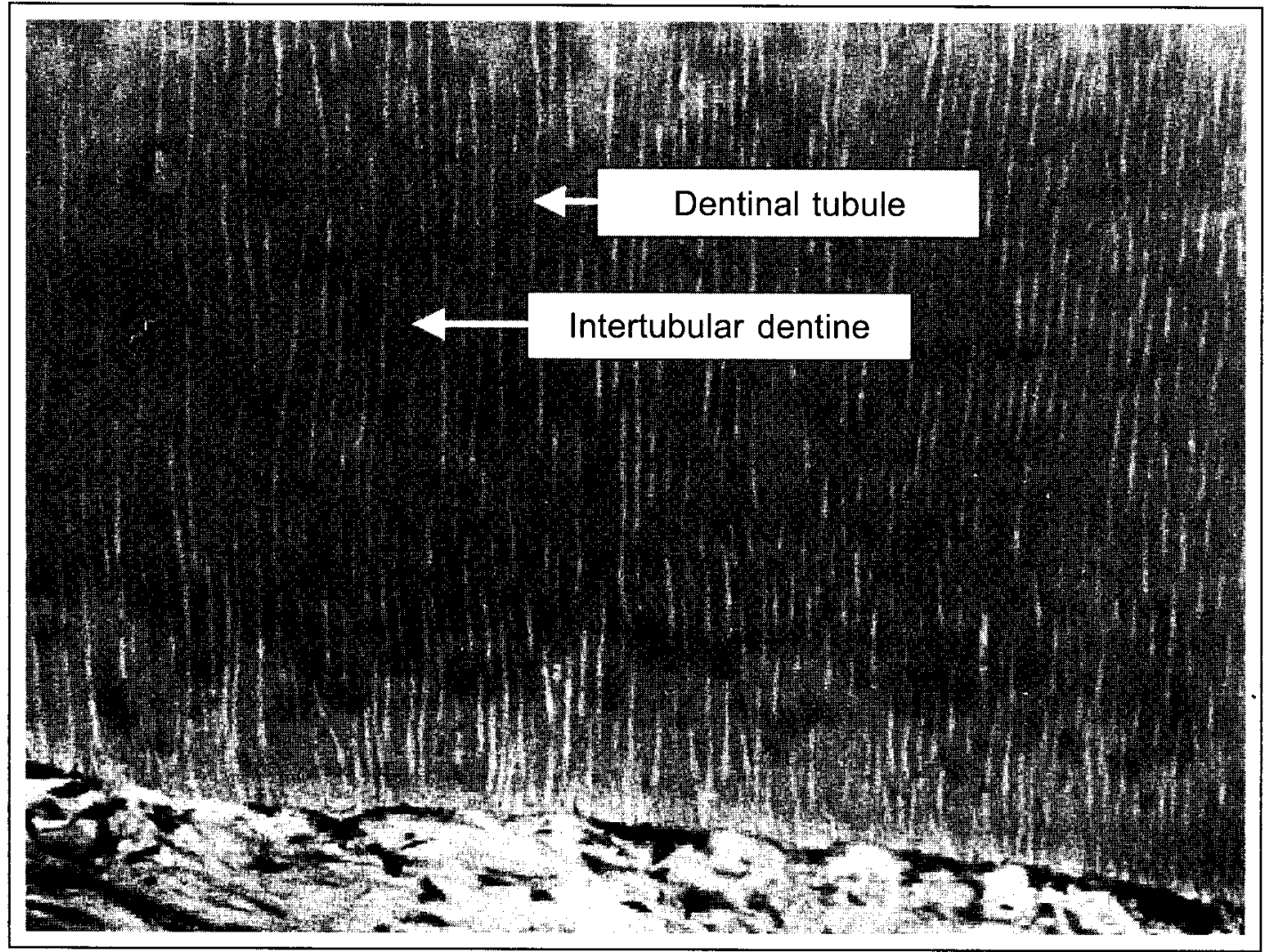

Figure 2: Gomori trichome stained section at zone 1. The intertubular dentine is clearly stained compared to the dentinal tubules. (X1O) 


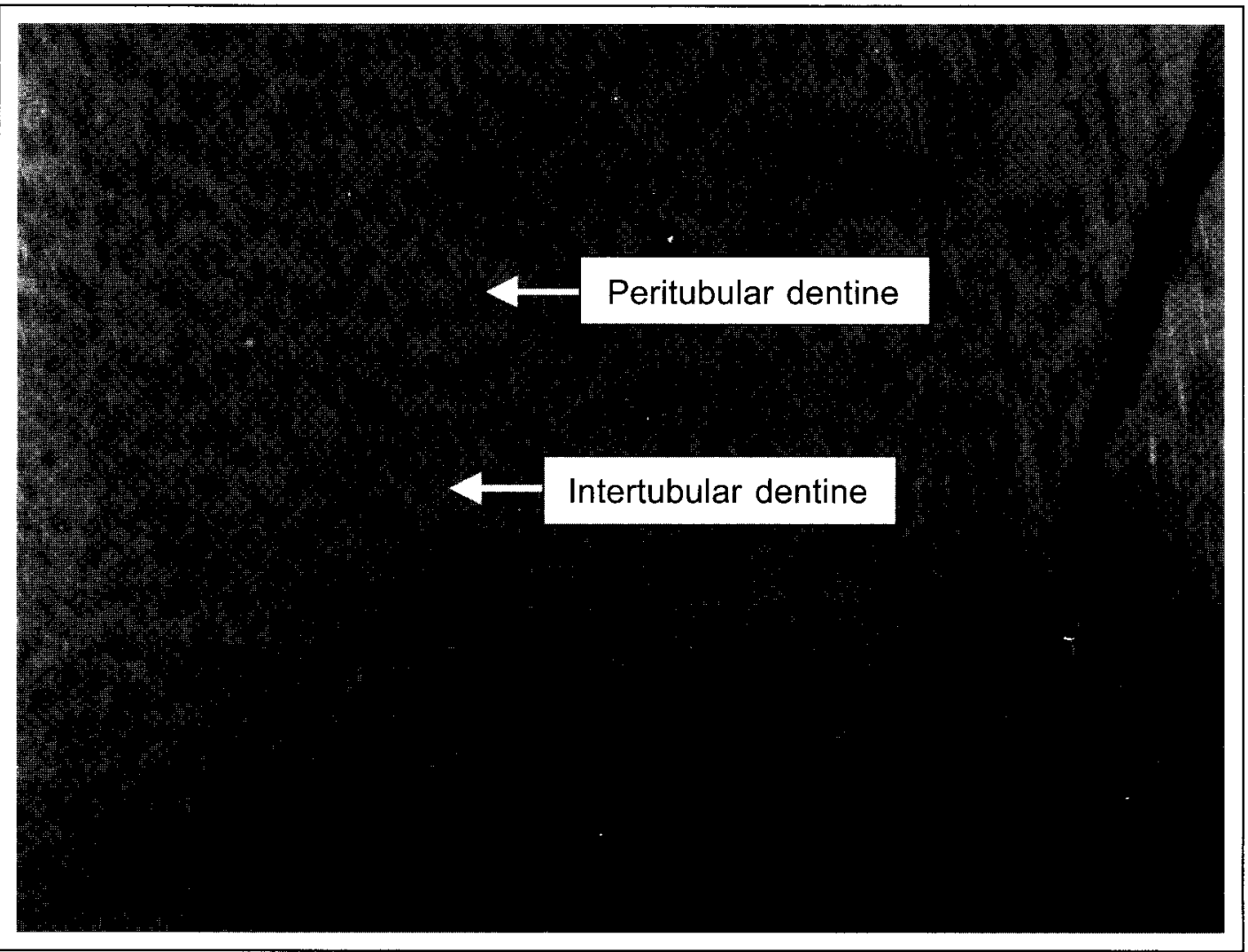

Figure 3: Gomori trichome stained section at zone 2. There is less staining of the intertubular dentine while the peritubular dentine is clearly stained at the periphery. (X40)

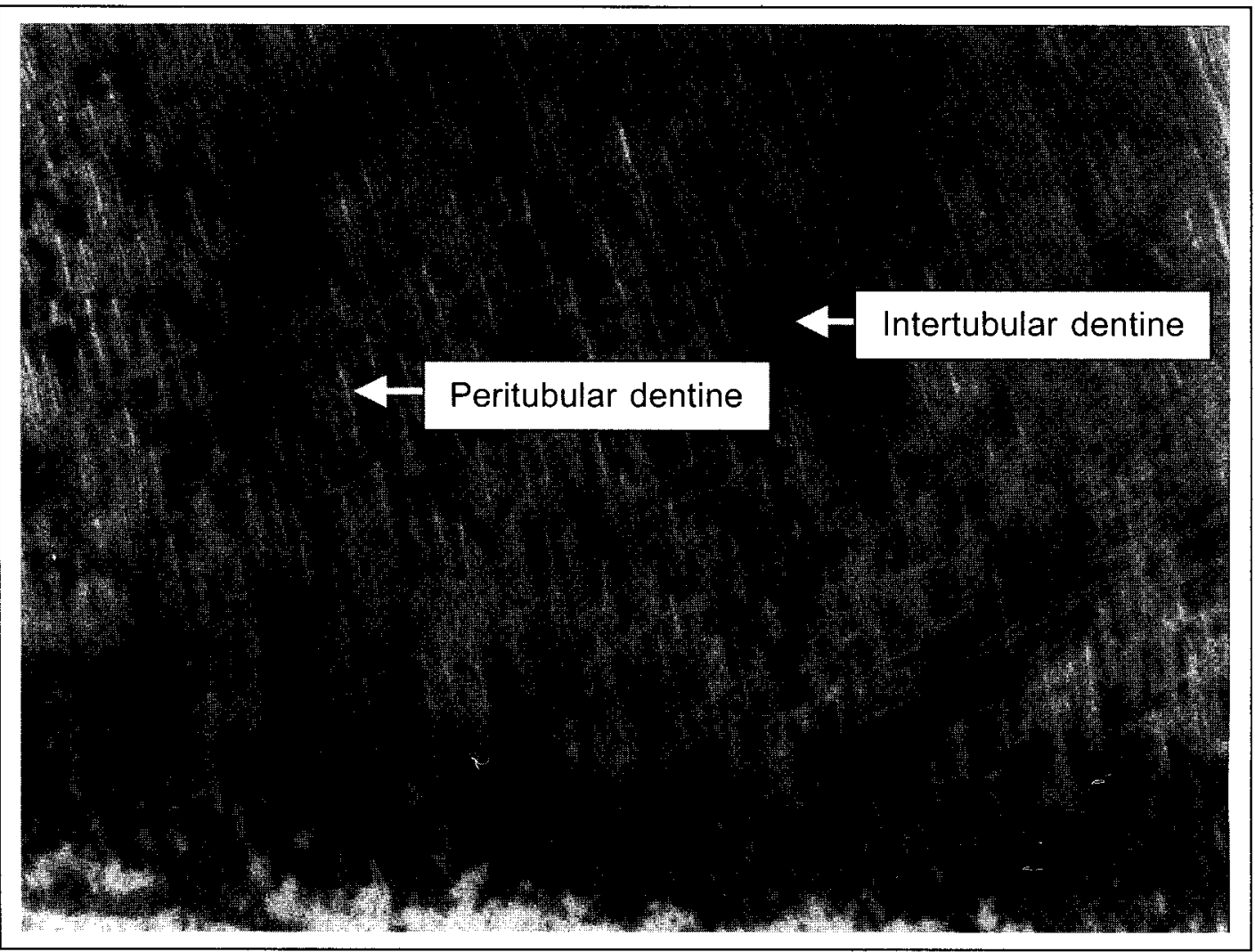

Figure 4: Gomori trichome stained section at zone 3 and 4. The intertubular dentine is similarly stained as in Fig. 3 while the staining of the peritubular dentine is completed. (X40) 
once again seems to indicate that this zone was rich in mucopolysaccharide acid which was linked to the calcification process of dentine.

This outer most zone appeared to be heavily stained and this may be due to the fact that in the outer zone, there are less tubules compared to the inner zone. Therefore the outer zone is made up of more intertubular dentine then peritubular dentine, giving it a more reddish colour.

\section{CONCLUSIONS}

It was shown that Gomori trichome produced 4 zonations of dentine. The strongly stained intertubular dentine in zone 1 and peritubular dentine in zon 3 seems to support the findings of other workers $(3,4,5)$ that the formation of intertubular dentine is taking place in zone 1 and peritubular dentine in zone 3 .

\section{ACKNOWLEDGEMENT}

The author wishes to acknowledge Encik Saifuddin bin Haji Abdul Razak for his assistance in preparation of the slides.

\section{REFERENCES}

1. Ritchie HH, Ritchie DG, Wang LH. Six decades of dentinogenesis research. Historical and prospective views on phosphoryn and dentine sialoprotein. Eur J Oral Sci 1998; 106 (Suppl 1): 211-20.

2. Linde A: Dentine and dentinogenesis, vols 1 and 2, 1984. CRC Press.

3. Goldberg M, Triller M, Escaig F, GenotelleSeptier D, Weill R. Acid mucopolysaccharide detection on ultra-thin sections by alcian blue in dental tissues embedded in Epon. J BioI Buccale 1976; 4: 155-64.
4. Mjor IA. Stainability of decalcified human coronal dentine. Arch. Oral BioI. 1966; 11: 1293-305.

5. Symons NBB. A histochemical study of the intertubular and peritubular matrices in normal human dentine. Arch. Oral BioI. 1961; 5: 24150 .

6. Allred H. The differential staining of peritubular and intertubular matrices in human dentine. Arch Oral BioI. 1968; 13: 1-11.

7. Ten Cate AR. Oral Histology: Development, Structure and Function, 5th edn. 1998. CV Mosby.

8. Weiner S, Veis A Bemiash E, Arad T, Dillon JW, Sabsay B, Siddiqui F. Peritubular dentine Formation: organization and the macromolecular constituents in human teeth. J Struct BioI 1999; 126: $27-41$.

9. Pearse A.G.E. Histochemistry. Theoretical and Applied. 2nd ed. 1961 London: Churchill.

10. Takagi M, Hishikawa H, hosokawa Y, Kagami A, Rahemtulla F. Immunohistochemicallocazation of glycosaminoglycans and proteoglycans in predentine and dentine of rat incisors. J Histochem Cytochem 1990; 38: 319-24.

11. Goldberg M, Septier DS. Differential staining of glycosaminoglycans in the predentine and dentine of rat incisor using cuprolinic blue at various magnesium chloride concentrations. Histochem J 1992; 24 : 648-54.

12. Goldberg M, Septier D. A comparative study of the transition between predentine and dentine, using various preparative procedures in the rat. Eur J Oral Sci 1996; 104(3): 269-77.

13. Blake GC. The peritubular translucent zones in human dentine. Brit. Dent. J. 1958; 104: 57-64.

14. Miller 1. The micro-radiographic appearance of dentine. Brit. Dent. 1. 1954; 97: 7-9. 